\title{
Elektronische Patientenakte ausreichend, aber ...
}

\begin{abstract}
- Nach einem Urteil des Oberlandesgericht (OLG) Naumburg sind elektronisch geführte Patientenakten alleine beweisfähig, auch dann, wenn nachträgliche Veränderungen möglich sind (Az.: 1 U 45/11). Im Streitfall war es bei einem Patienten im Rahmen einer Magenspiegelung zu einer Verletzung der Speiseröhre gekommen. Nach Aussage des gerichtlichen Sachverständigen lag zwar kein Schadensersatz begründender grober Behandlungsfehler vor, doch rügte der Patient die mangelnde Aufklärung über dieses Behandlungsrisiko. Der beweispflichtige Arzt verwies auf seine elektronische Patientenakte, die den Vermerk enthielt, dass der Patient im Vorfeld der Behandlung sehr wohl auf dieses Risiko hingewiesen wurde. Diese „Dokumentation" rügte der Patient als nicht ausreichend, da der Arzt den Eintrag auch im Nachhinein hätte erstellen können.
\end{abstract}

Nach Auffassung der Richter kann allerdings einer formell und materiell ordnungsgemäßen Dokumentation bis zum Beweis des Gegenteils Glauben geschenkt werden. Um die Vollständigkeit der Dokumentation zu erschüttern, müssten konkret erkennbare Anhaltspunkte vorliegen, beispielsweise das Vorliegen nachträglicher Änderungen oder eine Fertigung erst mit langem zeitlichem Abstand. Der Beweiswert einer ärztlichen Behandlungsdokumentation werde dadurch nicht gemindert, dass ein EDV-Programm verwendet wird, das nicht gegen nachträgliche Veränderbarkeit gesichert ist, wenn der Arzt plausibel darlegen kann, dass seine Eintragung richtig ist und sie aus medizinischen Gründen schlüssig erscheint.

\section{MMW Kommentar}

Der Richterspruch sollte allerdings in Verbindung mit einem Urteil des Bundesgerichtshofs (BGH) gesehen werden, wonach es bei Schadensersatzansprüchen des Patienten beim Grundsatz der Beweislastumkehr bleibt. Nicht der Patient muss beweisen, dass der Arzt einen groben Behandlungsfehler begangen hat. Vielmehr muss der Arzt beweisen, dass sein medizinisches (Fehl-) Verhalten nicht zu dem späteren Gesundheitsschaden bei dem Patienten geführt oder dazu beigetragen hat (Urteil vom 19. Juni 2012, AZ: VI ZR 77/11). Es ist deshalb nicht in allen Fällen ratsam, sich ausschließlich auf eine elektronische Patientenakte zu verlassen. Zumindest bei Eingriffsleistungen in der Praxis sollte man die einschlägigen Aufklärungsbögen verwenden und diese auch vom Patienten unterschreiben lassen.

\section{Hausärzteverband will keinen neuen EBM im Eilverfahren}

- Der Deutsche Hausärzteverband weist darauf hin, dass in der Vertreterversammlung der KBV (KBV-VV) am 6./7.12.2012 der Beschluss gefasst wurde, dass sowohl zum Konzept der hausärztlichen als auch zum Konzept der fachärztlichen Versorgungsebene mindestens ein gleichzeitiger Beschluss der KBV zu einer dauerhaften, stabilen und zukunftsweisenden Trennung der Gesamtvergütung notwendig ist. Darüber hinaus hätten die Mitglieder der KBV-VV beschlossen, das dort vorgestellte Konzept eines neuen EBM unter Beachtung der Stellungnahmen der Berufsverbände und der beratenden Fachausschüsse weiterzuentwickeln.

Diesem Beschluss sei bisher nicht Rechnung getragen worden, vielmehr habe der für die hausärztliche Versorgung zustän-

dige Berufsverband seit der genannten Vertreterversammlung keine Informationen mehr über die Weiterentwicklung des EBM erhalten und deshalb auch keine Stellungnahme abgeben können. Stattdessen sei es zu einem ersten Gespräch zwi-

\section{MMW Kommentar}

Der Hausärzteverband macht in seinem Schreiben deutlich, dass er keinen Alleingang bei der Neugestaltung des EBM im hausärztlichen Bereich akzeptiert, wenn gleichzeitig eine Neugestaltung des Trennungsfaktors vorgenommen werden soll und eine kostenneutrale Anhebung des Orientierungspunktwertes auf den Kalkulationspunktwert von 5,11 Cent. Er sieht die Gefahr, dass es bei einer Umsetzung dieser schen Vertretern der KBV und der Krankenkassen im Arbeitsausschuss des Bewertungsausschusses gekommen, ohne eine solche Stellungnahme abzuwarten bzw. in die Ausgestaltung des neuen EBM einfließen zu lassen.
Maßnahmen und einer erst späteren Neugestaltung des fachärztlichen EBM - wie die $K B V$ das vorhat - zu erheblichen finanziellen Verschiebungen zwischen dem haus- und dem fachärztlichen Honorarbereich zu Lasten der Hausärzte kommt. Betrachtet man das, was die KBV hier vorhat, ist diese Sorge des Hausärzteverbandes mehr als berechtigt. 